\title{
Low-temperature Glauber dynamics under weak competing interactions
}

\author{
M. D. Grynberg \\ Departamento de Física, Universidad Nacional de La Plata, 1900 La Plata, Argentina
}

(Received 26 December 2014; published 19 March 2015)

\begin{abstract}
We consider the low but nonzero-temperature regimes of the Glauber dynamics in a chain of Ising spins with first- and second-neighbor interactions $J_{1}, J_{2}$. For $0<-J_{2} /\left|J_{1}\right|<1$ it is known that at $T=0$ the dynamics is both metastable and noncoarsening, while being always ergodic and coarsening in the limit of $T \rightarrow 0^{+}$. Based on finite-size scaling analyses of relaxation times, here we argue that in that latter situation the asymptotic kinetics of small or weakly frustrated $-J_{2} /\left|J_{1}\right|$ ratios is characterized by an almost ballistic dynamic exponent $z \simeq 1.03(2)$ and arbitrarily slow velocities of growth. By contrast, for noncompeting interactions the coarsening length scales are estimated to be almost diffusive.
\end{abstract}

DOI: 10.1103/PhysRevE.91.032129

PACS number(s): 05.50.+q, 05.40.-a, 64.60.Ht, 75.78.Fg

\section{INTRODUCTION}

Processes usually referred to as phase-ordering dynamics, coarsening, or domain growth following an instantaneous quench from a high to a subcritical temperature-ordered phase have been the focus of intense research efforts over the past few decades [1]. At the same time, many interesting issues continue to arise in the field. An example of this is posed by the zero-temperature Glauber dynamics [2-4] of Ising models in which energy-increasing spin flips are rejected. As a result, in higher dimensions the dynamics is not always able to reach its ground states but rather probably gets trapped in metastable situations [4]. This lack of ergodicity may even appear in one dimension when competing interactions are considered [5]. In particular, this is the case of the one-dimensional (1D) axial next-nearest-neighbor Ising model [6,7] of spins $S= \pm 1$ and energy configurations

$$
E=-J_{1} \sum_{i} S_{i} S_{i+1}-J_{2} \sum_{i} S_{i} S_{i+2},
$$

in which frustration arises when combining antiferromagnetic (AF) $J_{2}$ couplings $\left(J_{2}<0\right)$ with $J_{1}$ exchanges of any sign [8]. From its original formulation in higher dimensions to account for (equilibrium) structure factors in rare earths along their single competing axes [9], this model has been recurrently investigated ever since $[7,10,11]$. When it comes to the dynamics, the magnetic relaxation of recently synthesized molecular chains with strong Ising anisotropy [12] was also considered in this framework by means of a decoupling approximation of the underlying master equation [13]. In contrast to previous decoupling schemes [14] and numerical simulations [15], it was found that in the strongly frustrated but ergodic regime $-J_{2}>\left|J_{1}\right|$, the Glauber relaxation time $\tau$ becomes of the order of the equilibrium correlation length $\xi$ [13]. Since at evolution times comparable with $\tau$ the average domain size is $\propto \xi$, this suggests that the ordering scale should coarsen ballistically (linearly in time) rather than in a diffusive [14] or subdiffusive manner [15].

Here we will not venture into that latter controversy but focus instead on the less studied situation of weakly competing ratios $r \equiv-J_{2} /\left|J_{1}\right|$ in the limit of $T \rightarrow 0^{+}$. In marked contrast to the usual Glauber dynamics $[2,3]\left(J_{2}=0\right)$ and irrespective of how small the frustration might be, note that at $T=0$ the length scale of magnetic domains does not coarsen [5]. This is because for $0<r<1 / 2$ (say, with $J_{1}>0$ and starting from a disordered phase), the ferromagnetic (F) state is unreachable as kinks or domain walls cannot approach each other less than two lattice spacings, thus precluding their annihilation via single-spin flips [5]. In turn, when $1 / 2<r<1$ there is no way to reach the fourfold degenerate ground state (consisting of consecutive pairs of oppositely oriented spins $\cdots \bullet \bullet \circ \circ \cdots)$, because the creation of kink pairs is forbidden at $T=0$ and so isolated domains of width $\geqslant 3$ cannot break up [5]. (These issues get clear with the aid of Table I, introduced in Sec. II.) In either case, the system rapidly ends up fluctuating in a metastable phase of domains of length $\geqslant 2$, in which kinks can wander around at no energy cost while keeping their density fixed $[5,15]$. In the thermodynamic limit this latter approaches a finite fraction $\rho=(5-\sqrt{5}) / 10$ [15], so large domain lengths $\lambda$ are distributed as $\rho e^{-\rho \lambda}[16]$ and the average domain size cannot but remain bounded as $\sim \int_{0}^{\infty} \lambda \rho e^{-\rho \lambda} d \lambda=1 / \rho$. As for $r=1 / 2$, there the ground state is accessible but highly degenerate, and its order range also turns out to be finite, i.e., $\xi=1 / \ln g$ with golden ratio $g=\frac{1}{2}(\sqrt{5}+1)[6,7]$.

Despite this noncoarsening scenario, in what follows we will consider the large time scaling effects introduced by a small but nonzero temperature in this weakly frustrated dynamics. Although at $T=0$ all AF strings disappear exponentially fast in time [5], and the local persistence (i.e., the probability for a spin to remain in its original state at a given time) decreases as a stretched exponential form [15], yet at infinitesimal temperatures the actual relaxation time gets arbitrarily large, just because of the energy barriers responsible of the mentioned metastable phase. In addition, once ergodicity is restored note that for $0<r<1 / 2$ and temperatures much below the so-called disorder line, $\cosh K_{1}=e^{2 K_{2}}[6,7,10]$, the now accessible equilibrium correlation length grows unbounded as $\sim \frac{1}{2} e^{\left(2 K_{1}+4 K_{2}\right)}$, while for $r>1 / 2$ it does so as $\sim 2 e^{\left|K_{1}+2 K_{2}\right|}$ [17], where $K_{i} \equiv J_{i} / k_{B} T$. Thus, following critical dynamic theories [18], in approaching $T=0^{+}$both of these growing space and time scales should behave as $\tau \propto \xi^{z}$ with a dynamic exponent $z$ characteristic of the universality class to which the dynamics belongs. In the long-time limit it basically describes how fast the coarsening scale is spreading and in a way that if spatial coordinates are rescaled as $x \rightarrow x / t^{1 / z}$ the probability distribution of domain lengths remains invariant [1]. On the other hand, in nearing the critical regime the correlation length becomes comparable to the system size $L$. Hence it is customary to 
TABLE I. (Color online) Glauber transition probabilities, energy changes, and symmetrized (S) nondiagonal matrix elements of the evolution operator transformed as in Eq. (9) for both kink pairing and diffusion processes under $J_{1}$ and $J_{2}$ interactions. Filled and empty circles denote original spins with opposite orientations in turn conforming kinks (vertical lines) on the dual chain. Upper and lower signs stand, respectively, for the forward $(\rightarrow)$ and backward $(\leftarrow)$ processes brought about by flipping central spins. All events are classified according to the projector types defined in Eq. (7).

\begin{tabular}{|c|c|c|c|c|c|}
\hline \multicolumn{2}{|l|}{ Pairing process } & \multirow{2}{*}{$\frac{\text { Rate }(\rightleftarrows)}{\frac{1}{2}(1 \pm \tanh P)}$} & \multirow{2}{*}{$\frac{\beta \Delta E(\rightleftarrows)}{\mp 2 P}$} & \multirow{2}{*}{$\frac{\text { S element }}{\frac{1}{2} \operatorname{sech} P}$} & \multirow{2}{*}{$\frac{\text { Projector }}{\mathcal{P}^{(1)}}$} \\
\hline$\circ \circ|\bullet| \circ \circ \quad$ & $\circ \quad 0 \quad 0 \quad 0 \quad 0$ & & & & \\
\hline$\bullet|\circ| \bullet \mid \circ \quad \circ \quad \leftleftarrows$ & - $\mid \begin{array}{llll}0 & 0 & 0 & 0\end{array}$ & $\frac{1}{2}\left(1 \pm \tanh 2 K_{1}\right)$ & $\mp 4 K_{1}$ & $\frac{1}{2} \operatorname{sech} 2 K_{1}$ & $\mathcal{P}^{(2)}$ \\
\hline$\circ \circ|\bullet| \circ \mid \bullet \leftleftarrows$ & 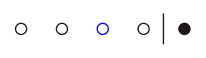 & $\frac{1}{2}\left(1 \pm \tanh 2 K_{1}\right)$ & $\mp 4 K_{1}$ & $\frac{1}{2} \operatorname{sech} 2 K_{1}$ & $\mathcal{P}^{(3)}$ \\
\hline$\bullet|\circ| \bullet|\circ| \bullet$ & - $\mid \begin{array}{lll}0 & 0 & 0\end{array}$ & $\frac{1}{2}(1 \pm \tanh Q)$ & $\mp 2 Q$ & $\frac{1}{2} \operatorname{sech} Q$ & $\mathcal{P}^{(4)}$ \\
\hline Diffusion process & & Rate $(\rightleftarrows)$ & $\beta \Delta E(\rightleftarrows)$ & S element & Projector \\
\hline - $\bullet \quad \bullet \circ \circ \quad$ & - -1000 & $1 / 2$ & 0 & $1 / 2$ & $\mathcal{P}^{(1)}$ \\
\hline$\circ|\cdot \bullet| 00 \quad$ & $\circ|\cdot| \begin{array}{lll}0 & 0 & 0\end{array}$ & $\frac{1}{2}\left(1 \pm \tanh 2 K_{2}\right)$ & $\mp 4 K_{2}$ & $\frac{1}{2} \operatorname{sech} 2 K_{2}$ & $\mathcal{P}^{(2)}$ \\
\hline$\circ \circ|\bullet \cdot| 0 \leftleftarrows$ & $\circ \quad 0 \quad 0|\bullet| 0$ & $\frac{1}{2}\left(1 \pm \tanh 2 K_{2}\right)$ & $\mp 4 K_{2}$ & $\frac{1}{2} \operatorname{sech} 2 K_{2}$ & $\mathcal{P}^{(3)}$ \\
\hline$\bullet|\circ| \bullet \cdot \mid 0 \quad$ & - $\mid$\begin{tabular}{ll|l|l|}
$\circ$ & $\circ$ & $\bullet$
\end{tabular} & $1 / 2$ & 0 & $1 / 2$ & $\mathcal{P}^{(4)}$ \\
\hline
\end{tabular}

think that the above scaling relation can be traded in practice for the finite-size behavior $\tau_{L} \propto L^{z}$, provided $L$ is taken sufficiently large [19]. Thus, in the following sections we attempt to provide an estimation of this exponent from the information of finite systems. To that aim, first we will recast the master equation [20] governing these Markov processes in terms of a quantum spin representation of the associated Glauber operator, i.e., the Liouvillian or evolution matrix of the problem [20]. This latter lends itself more readily for a finite-size scaling analysis of actual relaxation times as these are embodied in spectral gaps which we will subsequently evaluate by exact diagonalizations [21].

The layout of this work is organized as follows. Section II outlines the basic transition probability rates of these processes (all nonzero at $T>0$ ) using a kink or dual representation here thought of as a spin- $\frac{1}{2}$ dynamics. Exploiting detailed balance [20] the associated evolution operator can then be brought to a symmetric representation via simple nonunitary spin transformations. In part, this simplifies the numerical analysis of Sec. III in which spectrum gaps are obtained via standard recursive techniques [21] in various situations, both for competing and noncompeting interactions. This provides a sequence of finite-size approximants to dynamic exponents which are then combined with extrapolations [19,22] to partly remedy unavoidable size effects. We close with Sec. IV, which contains a recapitulation along with brief remarks on open issues and possible extensions of this work.

\section{STOCHASTIC DYNAMICS}

As is known [2,3], in the Glauber dynamics the heat bath is thought of as inducing fluctuations in the form of single-spin flip processes, thus causing the states $|S\rangle=\left|S_{1}, \ldots, S_{L}\right\rangle$ to change randomly. For instantaneous quenches the transition probability rates per unit time $W\left(S \rightarrow S^{\prime}\right)$ between two configurations $|S\rangle,\left|S^{\prime}\right\rangle$ (here differing in the state of one spin), are taken time independent and chosen to satisfy the detailed balance condition [20]

$$
P_{B}(S) W\left(S \rightarrow S^{\prime}\right)=P_{B}\left(S^{\prime}\right) W\left(S^{\prime} \rightarrow S\right), \forall|S\rangle,\left|S^{\prime}\right\rangle
$$

to enforce the system to relax towards the Boltzmann distribution $P_{B}(S) \propto e^{-\beta E(S)}$ at large times [20]. Hereafter temperatures are measured in energy units, or, equivalently, the Boltzmann constant in $\beta \equiv 1 /\left(k_{B} T\right)$ is set to 1 . For single-spin flip processes the simplest choice of rates complying with Eq. (2) corresponds to that of Glauber [1-3], which, adapted to Eq. (1), reads

$$
\begin{aligned}
W\left(S_{i} \rightarrow-S_{i}\right)= & \frac{\alpha}{2}\left\{1-S_{i} \tanh \left[K_{1}\left(S_{i-1}+S_{i+1}\right)\right.\right. \\
& \left.\left.+K_{2}\left(S_{i-2}+S_{i+2}\right)\right]\right\} .
\end{aligned}
$$

Here $\alpha^{-1}$ simply sets the time scale of the microscopic process and from now on is taken equal to unity. Just as frustration may occur irrespective of nearest-neighbor (NN) interactions being F or AF [8], note that the dynamics is not affected either by the nature of those couplings. To check this out consider, for instance, the mapping $S_{2 i} \rightarrow-S_{2 i}$, while leaving the odd spins unchanged. Assuming both periodic boundary conditions (PBC) and an even number of spins - henceforth considered throughout-then $S_{i} S_{i+1} \rightarrow-S_{i} S_{i+1}$ and $S_{i} S_{i+2} \rightarrow S_{i} S_{i+2}$. Therefore, by taking $J_{1} \rightarrow-J_{1}$ the rates of Eq. (3) are left invariant and so is the mapped dynamics because the single-spin flip process maps onto itself. (This reasoning, however, would not apply to a dynamics such as the Kawasaki one [1] as the mapping would then allow parallel NN spins to flip.) Thus, without lose of generality in what follows we shall consider, say, ferromagnetic NN interactions along with $J_{2}$ couplings of either sign. We shall get back to this point by the end of this section.

As is schematized in Table I, there are basically eight processes (some of them symmetric by reflection in the active spin), which we now disaggregate into two sets of dual events, namely pairing and diffusion of kinks. Also, after introducing the parameters

$$
P=2\left(K_{1}+K_{2}\right), \quad Q=2\left(K_{1}-K_{2}\right),
$$

we list the transition rates of these processes along with their energy gradients. Due to the latter, we note in passing that at $T=0$ and $0<r<1$ all AF domains would be irreversibly removed by pairing, while the remaining set of diffusion 
processes would maintain all kinks separated in at least two lattice spacings. (See the related discussion in Sec. III A.) This is in line with the metastable picture given in Sec. I, as it should, though that is to be contrasted with the actual nonzero low-temperature dynamics and towards which we next turn.

\section{A. Evolution operators}

The transition rates referred to in Eq. (3) form part of the so-called master equation [20] controlling the time evolution of these Markovian processes. It determines the probability distribution $|P(t)\rangle \equiv \sum_{S} P(S, t)|S\rangle$ to observe the system in one the above states $|S\rangle$ at a given time $t$. Following some of the formal analogies between stochastic and quantum systems [23] we now think of the master equation as a Schrödinger one in an imaginary time, that is,

$$
\frac{\partial}{\partial t}|P(t)\rangle=-H|P(t)\rangle,
$$

thus allowing us by formal integration to obtain the probability distribution at subsequent moments from the action of $H$ on a given initial condition, i.e., $|P(t)\rangle=e^{-H t}|P(0)\rangle$. Here, the "Hamiltonian" (not necessarily Hermitian in this context) or Liouvillian operator generating the dynamics is defined through the matrix elements

$$
\left\langle S^{\prime}|H| S\right\rangle= \begin{cases}-W\left(S \rightarrow S^{\prime}\right), & \text { for } \quad S \neq S^{\prime}, \\ \sum_{S^{\prime} \neq S} W\left(S \rightarrow S^{\prime}\right), & \text { for } \quad S=S^{\prime},\end{cases}
$$

which, by conservation of probability, constrain all columns to add up to zero. Thereby it can be shown $[20,24]$ that the steady state corresponds to a unique $H$ eigenmode with eigenvalue $\lambda_{0}=0$, whereas the relaxation time of any observable average $\sum_{S} \mathcal{O}(S) P(S, t)$ (such as the magnetization or equal-time pair correlations) is upper bounded by $1 / \operatorname{Re}\left(\lambda_{1}\right)>0$, with $\lambda_{1}$ being the first excitation level of the $H$ spectrum.

Since the phase-space dimension of these operators grows exponentially with the system size, an operational counterpart of Eq. (6) is needed to implement the recurrence techniques of Sec. III. On the other hand, to downsize at least part of those memory requirements, we now turn to a two-to-one mapping $\sigma_{i} \equiv-S_{i} S_{i+1}$ in which new Ising variables standing on dual locations denote the presence or absence of the kinks referred to above. As depicted in Table I, in that representation the Liouvillian must account for both of the process types brought about by flipping one of the originals $S_{i}$. In that regard, if we think of the states $\left|\sigma_{1}, \ldots \sigma_{L}\right\rangle$ as representing configurations of $\frac{1}{2}$-spinors (say in the $z$ direction), then the operational analog of Eq. (6) can be readily obtained with the aid of raising and lowering operators $\sigma^{+}, \sigma^{-}$. Clearly, the nondiagonal parts of $H$ should involve terms $\propto \sigma_{i-1}^{ \pm} \sigma_{i}^{ \pm}$ as well as other ones $\propto \sigma_{i-1}^{ \pm} \sigma_{i}^{\mp}$ associated, respectively, to the pairing and diffusion events sketched above (say, on dual locations $i-1, i$ ). However, note that those processes should be weighted by rates which actually depend on the kink occupation $\hat{n}=\sigma^{+} \sigma^{-}=\frac{1}{2}\left(1+\sigma^{z}\right)$ and vacancy $\hat{\mathrm{v}}=1-\hat{n}$ numbers of the nearest locations surrounding the active ones (see Table I). Thus, to reproduce the correlations associated to of each of these events, here we classify them according to projectors defined as

$$
\begin{aligned}
& \hat{\mathcal{P}}_{i}^{(1)}=\hat{v}_{i-2} \hat{v}_{i+1}, \quad \hat{\mathcal{P}}_{i}^{(2)}=\hat{n}_{i-2} \hat{v}_{i+1}, \\
& \hat{\mathcal{P}}_{i}^{(3)}=\hat{v}_{i-2} \hat{n}_{i+1}, \quad \hat{\mathcal{P}}_{i}^{(4)}=\hat{n}_{i-2} \hat{n}_{i+1},
\end{aligned}
$$

to which we assign in turn the variables $\left\{x_{1}, x_{2}, x_{3}, x_{4}\right\} \equiv$ $\left\{P, 2 K_{1}, 2 K_{1}, Q\right\} \quad$ and $\quad\left\{y_{1}, y_{2}, y_{3}, y_{4}\right\} \equiv\left\{0,2 K_{2},-2 K_{2}, 0\right\}$. Then it is straightforward to verify that the nondiagonal (nd) parts of the evolution operator accounting for those correlated pairing and diffusion rates are each given by

$$
\begin{aligned}
& H_{\mathrm{nd}}^{\text {(pair) }}=-\sum_{i} \sum_{j} \hat{\mathcal{P}}_{i}^{(j)}\left[f\left(x_{j}\right) \sigma_{i-1}^{-} \sigma_{i}^{-}+f\left(-x_{j}\right) \sigma_{i}^{+} \sigma_{i-1}^{+}\right], \\
& H_{\mathrm{nd}}^{(\mathrm{diff})}=-\sum_{i} \sum_{j} \hat{\mathcal{P}}_{i}^{(j)}\left[f\left(y_{j}\right) \sigma_{i-1}^{+} \sigma_{i}^{-}+f\left(-y_{j}\right) \sigma_{i}^{+} \sigma_{i-1}^{-}\right],
\end{aligned}
$$

where $f(u) \equiv \frac{1}{2}(1+\tanh u)$.

Now we can exploit detailed balance to readily bring these expressions into symmetric operators. For that purpose it suffices to consider the kink energies $E_{\sigma}=\sum_{i}\left(J_{1} \sigma_{i}-\right.$ $\left.J_{2} \sigma_{i} \sigma_{i+1}\right)$ associated to Eq. (1) with which we construct the diagonal nonunitary similarity transformation $\mathbb{D}|\sigma\rangle \equiv$ $e^{\frac{\beta}{2} E_{\sigma}}|\sigma\rangle$. Therefore, under this latter, the generic nondiagonal elements of Eq. (6) transform as

$$
W\left(\sigma \rightarrow \sigma^{\prime}\right) \rightarrow e^{\frac{\beta}{2}\left(E_{\sigma^{\prime}}-E_{\sigma}\right)} W\left(\sigma \rightarrow \sigma^{\prime}\right) .
$$

Since in the kink representation the $\sigma$ rates also comply with the detailed balance condition (2), then clearly these transformed nondiagonal elements become symmetric under $\mathbb{D}$. Identifying this diagonal operator simply with $\exp \left[\frac{1}{2} \sum_{i}\left(K_{1} \sigma_{i}^{z}-K_{2} \sigma_{i}^{z} \sigma_{i+1}^{z}\right)\right]$, then the above pairing and hopping terms will transform, respectively, as

$$
\begin{aligned}
\sigma_{i-1}^{ \pm} \sigma_{i}^{ \pm} & \rightarrow \exp \left[\mp K_{2}\left(\sigma_{i-2}^{z}+\sigma_{i+1}^{z}\right) \pm 2 K_{1}\right] \sigma_{i-1}^{ \pm} \sigma_{i}^{ \pm}, \\
\sigma_{i-1}^{ \pm} \sigma_{i}^{\mp} & \rightarrow \exp \left[ \pm K_{2}\left(\sigma_{i-2}^{z}-\sigma_{i+1}^{z}\right)\right] \sigma_{i-1}^{ \pm} \sigma_{i}^{\mp},
\end{aligned}
$$

(compare with symmetrized elements of Table I), while the diagonal projectors of Eq. (7) remain unchanged. Thus, after some algebraic manipulations we are finally led to the symmetric counterparts of Eqs. (8a) and (8b), namely

$$
\begin{aligned}
\mathcal{H}_{\mathrm{nd}}^{\text {(pair })}= & -\frac{1}{4} \sum_{i}\left[A_{+}+B\left(\sigma_{i-2}^{z}+\sigma_{i+1}^{z}\right)+A_{-} \sigma_{i-2}^{z} \sigma_{i+1}^{z}\right] \\
& \times\left(\sigma_{i-1}^{+} \sigma_{i}^{+}+\text {H.c. }\right), \\
\mathcal{H}_{\mathrm{nd}}^{(\mathrm{diff})}= & -\frac{1}{4}\left(1+\operatorname{sech} 2 K_{2}\right) \sum_{i}\left(1+\tanh ^{2} K_{2} \sigma_{i-2}^{z} \sigma_{i+1}^{z}\right) \\
& \times\left(\sigma_{i-1}^{+} \sigma_{i}^{-}+\text {H.c. }\right),
\end{aligned}
$$

where the $A_{ \pm}$and $B$ coefficients are given by

$$
\begin{aligned}
A_{ \pm} & =\frac{1}{2}(\operatorname{sech} Q+\operatorname{sech} P) \pm \operatorname{sech} 2 K_{1}, \\
B & =\frac{1}{2}(\operatorname{sech} Q-\operatorname{sech} P) .
\end{aligned}
$$

When it comes to the diagonal terms of Eq. (6), in the kink representation these basically count the number of ways in which a given configuration can access to other ones either by 
NN pairing or hopping. To probe and weight these attempts with the rates of Table I we resort once more to the projectors and number operators referred to in Eq. (7) and in terms of which the diagonal $(d)$ parts associated to those two type of processes can be summarized each as

$$
\begin{aligned}
& H_{d}^{(\text {pair })}=\sum_{i} \sum_{j} \hat{\mathcal{P}}_{i}^{(j)}\left[f\left(x_{j}\right) \hat{n}_{i-1} \hat{n}_{i}+f\left(-x_{j}\right) \hat{v}_{i-1} \hat{v}_{i}\right] \\
& H_{d}^{\text {(diff) }}=\sum_{i} \sum_{j} \hat{\mathcal{P}}_{i}^{(j)}\left[f\left(y_{j}\right) \hat{v}_{i-1} \hat{n}_{i}+f\left(-y_{j}\right) \hat{n}_{i-1} \hat{v}_{i}\right] .
\end{aligned}
$$

Evidently, by construction $\mathcal{H}_{d} \equiv H_{d}^{\text {(pair) }}+H_{d}^{\text {(diff) }}$ remains invariant under $\mathbb{D}$, thus collecting all projector contributions and defining further coefficients,

$$
\begin{aligned}
& C_{ \pm}=\frac{1}{2}(\tanh Q+\tanh P) \pm \tanh 2 K_{1}, \\
& D_{ \pm}=\frac{1}{2}(\tanh Q-\tanh P) \pm \tanh 2 K_{2},
\end{aligned}
$$

we are ultimately left with diagonal terms in a uniform field, along with two- and three-body interactions of the form

$$
\begin{aligned}
\mathcal{H}_{d}= & \frac{1}{4} \sum_{i}\left[\left(C_{+}+D_{-} \sigma_{i+1}^{z}+D_{+} \sigma_{i+2}^{z}\right) \sigma_{i}^{z}\right. \\
& \left.+\frac{1}{2} C_{-}\left(\sigma_{i-1}^{z}+\sigma_{i}^{z}\right) \sigma_{i-2}^{z} \sigma_{i+1}^{z}\right]+\frac{L}{2} .
\end{aligned}
$$

Together with Eqs. (11a) and (11b), this latter result completes the construction of the operational analog of Eq. (6). It is worth noting that for $J_{2}=0$ all many-body couplings disappear $\left(C_{-}=D_{ \pm}=0\right)$ along with both corrrelated pairing and hopping terms $\left(A_{-}=B=0\right)$, so $\mathcal{H}_{d}+\mathcal{H}_{\text {nd }}^{\text {(pair) }}+\mathcal{H}_{\text {nd }}^{\text {(diff) }}$ fully recovers the bilinear form of Ref. [3]. In that case the evolution operator can be diagonalized exactly, and its spectrum reduces to the $2^{L-1}$ manners of filling a band of elementary fermionic excitations $E_{q}=1-\tanh 2 K_{1} \cos q$ using an even number of Fourier moments $q \in\{ \pm \pi / L, \pm 3 \pi / L, \ldots$, $\pm(L-1) \pi / L\}$ [3]. Thereby, in the limit of $T \rightarrow 0$ the dynamics is typically diffusive as the inverse gap or relaxation time becomes $\propto L^{2}$. For $J_{2} \neq 0$, however, the problem is no longer soluble by analytic treatments but parity conservation still holds. Thus, in Sec. III we shall restrict the numerical analysis to states having only an even number of domain walls, as is natural when PBC are set in the original spin system.

As for the dynamics invariance with respect to the sign of $J_{1}$ referred to earlier in this section, consider for that matter the global spin rotation

$$
\vec{\sigma} \rightarrow R \vec{\sigma} R^{-1}, \quad R=\exp \left(-i \frac{\pi}{2} \sigma^{x}\right),
$$

under which $\sigma_{j}^{z} \rightarrow-\sigma_{j}^{z}$ and $\sigma_{j}^{ \pm} \rightarrow \sigma_{j}^{\mp}$. Hence, the effect of this similarity transformation on Eqs. (11a) and (15) is tantamount, respectively, to the substitutions $B \rightarrow-B$ and $C_{ \pm} \rightarrow-C_{ \pm}$, while leaving Eq. (11b), $A_{ \pm}, D_{ \pm}$, as well as the whole spectrum of the evolution operator unaltered. But recalling the definitions of $P$ and $Q$ [Eq. (4)] entering in these coefficients [Eqs. (12) and (14)], it is then clear that this equivalent description here would just merely correspond to the substitution $J_{1} \rightarrow-J_{1}$, as previously argued on more intuitive grounds.

Let us finally comment that also three-body interactions correlated with single-spin flips already appear at the level of the Glauber rates of Eq. (3). Specifically, exploiting basic relations of the hyperbolic functions, and using the above $C_{ \pm}$ and $D_{ \pm}$coefficients, it can be readily shown that those rates actually deploy terms of the form

$$
\begin{aligned}
W\left(S_{i} \rightarrow-S_{i}\right)= & \frac{1}{2}-\frac{S_{i}}{8}\left[\left(S_{i-1}+S_{i+1}\right)\left(C_{+}+C_{-} S_{i-2} S_{i+2}\right)\right. \\
& \left.-\left(S_{i-2}+S_{i+2}\right)\left(D_{+}+D_{-} S_{i-1} S_{i+1}\right)\right],(17)
\end{aligned}
$$

which in turn would yield up to four-body interactions in the original spin evolution operator.

\section{NUMERICAL RESULTS}

Armed with the contributions of Eqs. (11a), (11b), and (15) operating on a generic kink state $|\sigma\rangle$, we next turn to the exact evaluation of spectral gaps in finite systems within lowtemperature regimes. As a consistency check, first we verified that transforming the Boltzmann distribution $\left|P_{B}\right\rangle$ with the above diagonal $\mathbb{D}$,

$$
\left|\psi_{0}\right\rangle=\sqrt{\mathcal{Z}} \mathbb{D}\left|P_{B}\right\rangle=\frac{1}{\sqrt{\mathcal{Z}}} \sum_{\sigma} e^{-\frac{\beta}{2} E_{\sigma}}|\sigma\rangle,
$$

produces, in fact, by construction, the ground state of $\mathcal{H}_{\text {nd }}^{\text {(pair) }}+$ $\mathcal{H}_{\text {nd }}^{\text {(diff) }}+\mathcal{H}_{d}$ with eigenvalue $\lambda_{0}=0$ (the kink partition function $\mathcal{Z}$ here acting merely as a normalization factor). This also served to start up a Lanczos algorithm [21] with random initial states but chosen orthonormal to that equilibrium $\left|\psi_{0}\right\rangle$. In turn, the states generated by the Lanczos recursion also were subsequently reorthonormalized to $\left|\psi_{0}\right\rangle$. Thereafter, we obtained the first excited eigenmodes of the evolution operator using periodic chains of up to 24 sites (the main limitation for that being the $2^{L-1}$ dimensions of the kink space). Also, as a further preliminary test we retrieved the gap $\lambda_{1}\left(K_{1}, 0\right)=2\left(1-\tanh 2 K_{1} \cos \frac{\pi}{L}\right)$ of the standard Glauber dynamics [3], and checked out the aforementioned symmetry $\lambda_{1}\left(K_{1}, K_{2}\right)=\lambda_{1}\left(-K_{1}, K_{2}\right)$.

Besides length limitations, another restrictive issue to point out here is that for $J_{2}<0$ the Lanczos convergence slows down progressively as temperature is lowered because, as we shall argue and corroborate in a moment, the spacing of low-lying levels gets arbitrarily small even in finite chains. Due to the smallness of those gaps at least quadruple precision is needed, but for $r=-J_{2} /\left|J_{1}\right| \gtrsim 0.3$ and temperatures below $T /\left|J_{1}\right| \sim$ 0.05 the pace of convergence becomes impractical for the larger sizes at hand. Thus, we shall content ourselves with providing results within the weakly frustrated region $0<r \leqslant$ 0.3 , where nonetheless a clear universal trend already shows up. By contrast, for noncompeting interactions the spectral gaps of finite systems remain finite even in the limit of $T \rightarrow 0$ (alike the usual case of $J_{2}=0$ ), and the Lanczos convergence is faster. However, it will turn out that there are three types of behavior to examine depending on whether $-1<r<0, r=$ -1 , or $r<-1$. 


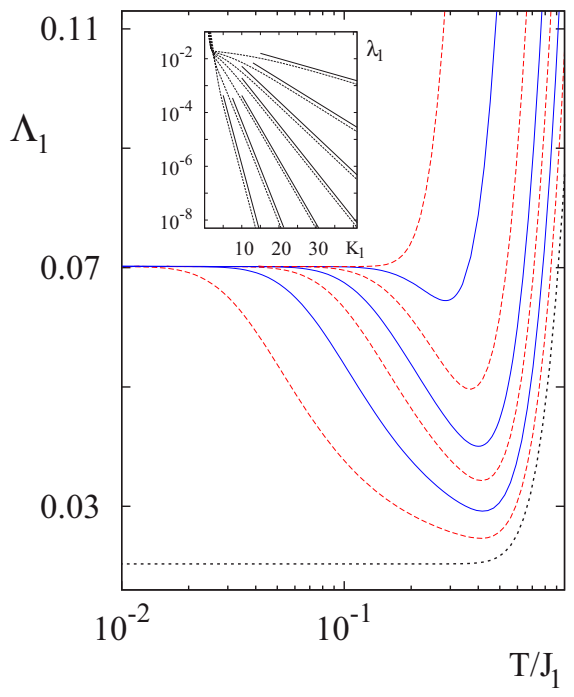

FIG. 1. (Color online) Normalized spectral gaps $\Lambda_{1}=e^{4 r K_{1}} \lambda_{1}$ with frustrated interactions for $L=22$ on approaching lowtemperature regimes and taking $r=0.3,0.2,0.1375,0.1,0.075$, $0.05,0.025$ (from top to bottom, alternating between dashed and solid lines). All $\Lambda$ 's saturate towards a common value $\propto L^{-z}$ (see Fig. 2). For comparison, the lowermost dotted curve denotes the soluble case $r=0$. The straight solid lines in the inset, closely following the asymptotic behavior of the corresponding data, are fitted with slopes $-4 r$.

\section{A. Weak competing interactions}

As noted in Sec. I, for $0<r<1$ the $T=0$ dynamics rapidly leaves the system in a metastable state of wandering kinks separated by at least two lattice spacings [5,15]. At low but nonzero temperatures, however, we see from Table I that for $0<r<1 / 2$ the energy barrier $\left(4\left|J_{2}\right|\right)$ responsible for that restriction corresponds to that of the second and third diffusion processes. But now those barriers can be surmounted provided time scales comparable to $2 /\left(1-\tanh 2\left|K_{2}\right|\right) \sim e^{4 r K_{1}}$ are considered. As a result, all ferromagnetic domains then rapidly coalesce just following the energy gradient $-4\left(J_{1}-\left|J_{2}\right|\right)<0$ of the first pairing process depicted above. Therefore it is reasonable to expect that even in a finite system the relaxation time to equilibrium should diverge as $e^{4 r K_{1}}$.

In Fig. 1 we test this hypothesis for several values of $r \in(0,0.3]$ as temperature is lowered. The saturation trends of the "normalized" gaps $\Lambda_{1} \equiv e^{4 r K_{1}} \lambda_{1}$ evidently confirm this expectation in this weakly frustrated region. This is also evidenced by the $-4 r$ slopes shown in the inset which closely follow those plain gap decays. Note that even a slight deviation from these slope values would result in strong departures from the saturation regimes exhibited in the main panel. In that temperature limit each $\Lambda_{1}$ appears to be independent of $r$, although they all differ from the usual Glauber gap $\simeq \pi^{2} / L^{2}$ of finite systems at $T=0$, thus already signaling a discontinuous behavior at $r=0$ (see also Sec. III B). In fact, and, more importantly, there the dynamic exponents are no longer diffusive, as indicated in Fig. 2 by the data collapse towards larger sizes. This was attained upon choosing an exponent $z \sim 1.1$, in turn consistent with the slopes read off from the inset of Fig. 2(a) where the finite-size behavior of the plain gap is exhibited at various temperatures. Analogous
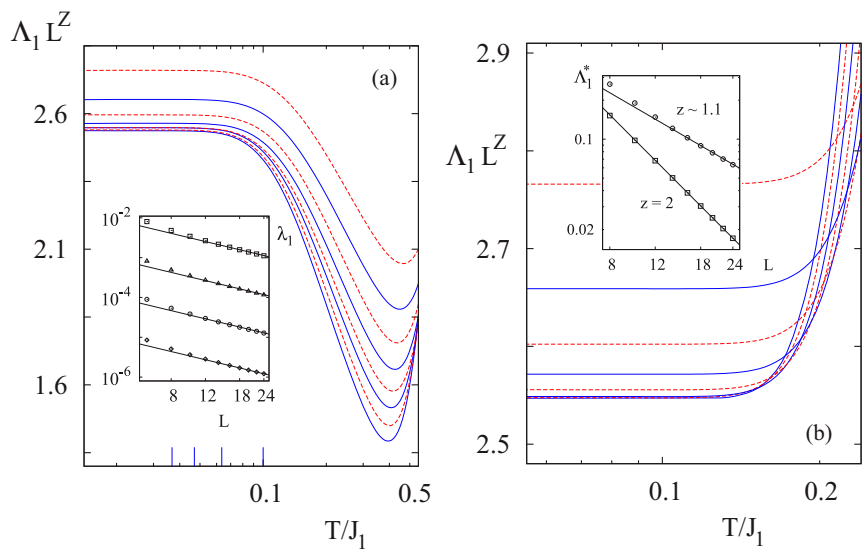

FIG. 2. (Color online) Finite-size scaling of normalized spectral gaps $\Lambda_{1}=e^{4 r K_{1}} \lambda_{1}$ for (a) $r=0.1$ and (b) $r=0.3$. In the main panels, sizes increase downwards as $L=2 k$ with $5 \leqslant k \leqslant 12$ alternating between dashed and solid lines. At low temperatures the data collapse of larger sizes was attained upon setting a common dynamic exponent $z \sim 1.1$ (however, see extrapolations of Sec. III C). The inset of (a) shows the plain spectral gap behavior for the temperatures indicated by vertical lines below, always consistent with an $\sim L^{-1.1}$ decrease. Similarly, the inset of (b) exhibits the typical finite-size decay of $\Lambda_{1}$ at saturation [Eq. (19)] for all $r$ 's considered in Fig. 1. For comparison, the diffusive case of $J_{2}=0$ is also shown here.

results were obtained for other weakly competing ratios, always recovering similar $z$ values, and, as mentioned above, yielding only size-dependent $\Lambda_{1}$ 's at saturation regimes. It seems then natural to put forward the hypothesis that in the limit of $T \rightarrow 0^{+}$and large $L$ these normalized gaps should all scale as

$$
\Lambda_{1}^{*}(L) \equiv \lim _{T \rightarrow 0^{+}} e^{-4 K_{2}} \lambda_{1}\left(K_{1}, K_{2}, L\right) \propto L^{-z},
$$

the proportionality factor just being a constant of the order of $\sim 2.55$, as observed in the main panels of Fig. 2 .

In the inset of Fig. 2(b) we check this out for several values of $r \in(0,0.3]$, in all cases using temperatures where the saturation trends were already settled. There the height of each data symbol encapsulates the results obtained for every $r$ in that range. In fact, the latter could be extended up to $r=0.4$ although, due to the convergence slowing mentioned above, no results are in our disposal for $L \geqslant 22$. Yet the key issue here is that no matter how weak the frustration might be, our analysis yields a dynamic exponent far from the diffusive $z=2$ obtained for $r=0$ (also shown for comparison). Let us anticipate that the nearly ballistic exponent $(z \simeq 1)$ to arise in Sec. III C from extrapolations to the thermodynamic limit also enables us to think of the large amplitudes of Eq. (19) as small asymptotic velocities of growth. Specifically, recalling that at times of order $1 / \lambda_{1}$ both the average domain size and correlation length become comparable to the system size, clearly from Eq. (19) it then follows that the ordering scale must coarsen almost linearly in time with an arbitrarily slow velocity $\propto e^{-4 r K_{1}}$.

It would be desirable to complete the weakly frustrated picture in the region $1 / 2<r<1$ but there, once more, the 


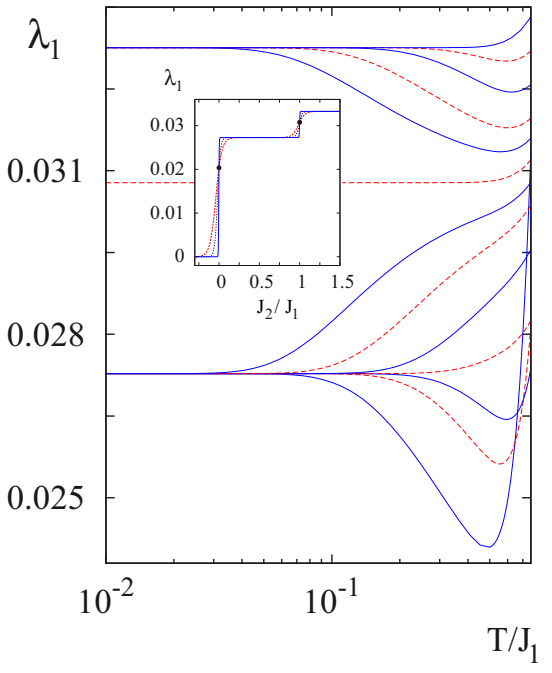

FIG. 3. (Color online) Gaps of noncompeting chains with $L=$ 22. From top to bottom the main panel depicts the branches corresponding, respectively, to (i) $-r=J_{2} /\left|J_{1}\right|>1 \quad(2,1.35,1.2$, 1.1, 1.05), (ii) $r=-1$, and (iii) $0<-r<1 \quad(0.95,0.9,0.8,0.6$, $0.3,0.2,0.1$ ), alternating between solid and dashed lines. Alike the frustrated regime (Fig. 1), at low temperatures each branch saturates towards common values $\propto L^{-z}$ (see Fig. 4). The inset exhibits the discontinuous tendency of these latter as temperature is lowered $\left(T / J_{1}=0.15,0.07,0.01\right)$. Filled circles stand at the intersection of cases $r=0$ and $r=-1$. Gaps at the leftmost regime (weakly competing, $r>0$ ) decay as $e^{-4 r K_{1}}$ (see inset of Fig. 1).

smallness of spectral gaps precluded convergence in the larger chains (here increasing by four sites to match the ground-state periodicity). As before, the gap turned out to decrease as $e^{-4 r K_{1}}$, though as temperature was lowered the relenting convergence pace impeded us to obtain further results for $L \geqslant 20$.

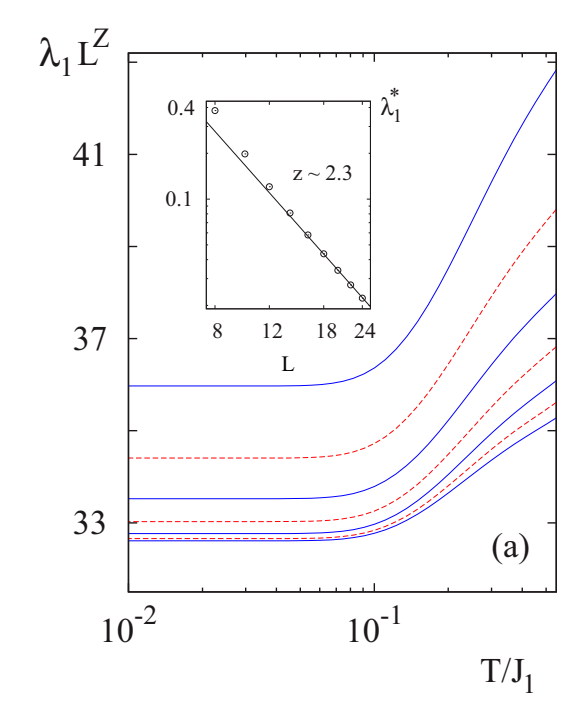

\section{B. Nonfrustrated regime}

Next we move on to the case of noncompeting interactions, i.e., $J_{2}>0$, for which there are no longer energy barriers hindering the access to low-temperature equilibrium. Thus, the relaxation times of finite systems remain bounded even at $T=0$. However, in that temperature limit it can be seen that a discontinuity already appears at the level of transition rates both at $r=0$ and $r=-1$. In the first case this affects either the second or third diffusion process of Table I, whereas for $r=-1$ it appears either in the first or fourth pairing process quoted there. Correspondingly, these discontinuities also affect the diagonal elements of the evolution operator via the $C_{ \pm}$and $D_{ \pm}$coefficients defined in Eq. (14) and are ultimately reflected in the gaps at low temperatures shown in Fig. 3. Its main panel clearly distinguishes different regimes below, at, and above $r=-1$, each encompassing a branch of spectral gaps with a common saturation point in the low-temperature limit. The inset of Fig. 3 displays the stepwise trend of this latter for both noncompeting and competing ratios, while a similar step pattern is followed by other low-lying $\lambda$ levels (not shown). Note here that in nearing $T=0^{+}$the left discontinuity at $J_{2}=0$ is signaling the abrupt crossover from the nondiffusive kinetics studied in Sec. III A to the usual Glauber dynamics with no energy barriers.

To characterize the nonfrustrated dynamics at large times, as before, we resort to a finite-size scaling analysis of these gaps in each of the above regimes. This we do in the main panels of Fig. 4 which exhibit the data collapse onto larger sizes obtained at and near $r=-1$. As might be expected from Fig. 3, the dynamic exponents producing these collapses turn out to be branch dependent only. In each case their values, all subdiffusive, can also be read off from the slopes that fit the finite-size decay of the corresponding gaps at low temperatures, now converging much faster than those of the case $r>0$. This is displayed in the insets of Fig. 4 where each data point is also representative of all $r$ 's studied
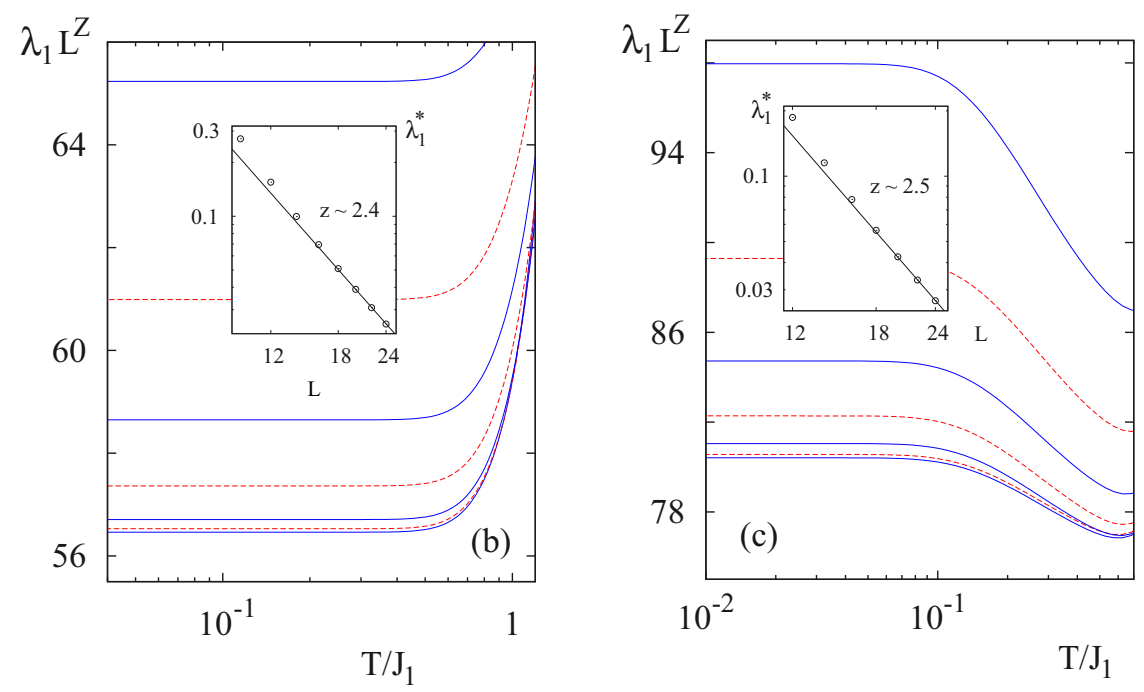

FIG. 4. (Color online) Typical finite-size scaling of gaps for nonfrustrated coupling regimes (Fig. 3), taking (a) $J_{2} /\left|J_{1}\right|=0.9$, (b) 1 , and (c) 1.1. Lengths, alternating between solid and dashed lines, increase downwards as $L=2 k$ with $6 \leqslant k \leqslant 12$. At low temperatures the data collapse of larger sizes in each branch was obtained upon choosing $z \sim 2.3,2.4$, and 2.5, respectively. In turn, these values are used as slopes to fit the typical finite-size decay of these gaps at saturation (denoted by $\lambda_{1}^{*}$ in the insets). See, however, extrapolations of Sec. III C. 
in the saturation regimes of Fig. 3. In particular, the gaps shown in the last inset are also typical of several coupling ratios which we studied additionally in the region $-r \gg 1$. However, the proximity of the resulting exponents calls into question whether these actually stem from different asymptotic dynamics per branch or are rather merely products of our finite-size limitations. In that latter respect, and aiming to observe in more detail those size effects, we now turn to standard extrapolations $[19,22]$ which go a step further than the collapse trends of Figs. 2 and 4.

\section{Extrapolations}

Having evaluated the spectral gaps of the evolution operator on finite sizes, an improved estimation of its dynamic exponents along with a measure of their convergence can be obtained upon considering the sequence of effective approximants defined as

$$
Z_{L}=\frac{\ln \left[\lambda_{1}^{*}(L) / \lambda_{1}^{*}(L-2)\right]}{\ln [(L-2) / L]} .
$$

These are simply the local slopes of the insets in Figs. 2 and 4, thus representing successive estimates of the gap closing in the low-temperature limit. Here all $L$ lengths are even to allow for the $J_{1} \rightarrow-J_{1}$ symmetry referred to above. Near criticality $\left(T=0^{+}\right)$this sequence of approximants is generally assumed to converge logarithmically $[19,22]$ as

$$
Z_{L}=z+\alpha_{1} L^{-a_{1}}+\alpha_{2} L^{-a_{2}}+\cdots,
$$

with $\alpha$ constants and $a$ exponents taken such that $0<a_{1}<$ $a_{2}<\cdots$. To minimize the number of fitting quantities we keep only the leading-order term of this expansion which just leave us with a nonlinear least-squares fit of three parameters. The results of this are depicted in Fig. 5, which summarizes the trends of these sequences across the situations considered in Secs. III A and III B. As anticipated, for weak competing interactions the extrapolated exponents thus resulting from these nonlinear regressions turn out to be almost ballistic $(z=$ 1 ), while those arising from the noncompeting regimes become slightly subdiffusive $(z>2)$, specifically

$$
z \simeq\left\{\begin{array}{l}
1.03 \pm 0.02, \text { for } 0<r \lesssim 0.3 \\
2.05 \pm 0.03, \text { for } 0<-r<1 \\
2.07 \pm 0.04, \text { for } r=-1 \\
2.08 \pm 0.03, \text { for } r<-1
\end{array}\right.
$$

In each case the degree of convergence of the corresponding sequences is similar (see the $a$ exponents mentioned in Fig. 5) but slower than that obtained in the standard dynamics of $r=0$ (also shown for comparison) and where the extrapolated $z$ exponent $\simeq 1.997 \pm 0.004$ comes out pretty close to its actual value.

We also fitted these sets of finite-size results using sequence transformation methods such as Vanden Broeck-Schwartztype ones [25], which yield basically analogous $z$ 's within the above margins of error. However, since in practice it is never really clear whether the assumed asymptotic behavior is sufficiently well realized by the data available [19], the slight differences among these subdiffusive exponents might well be

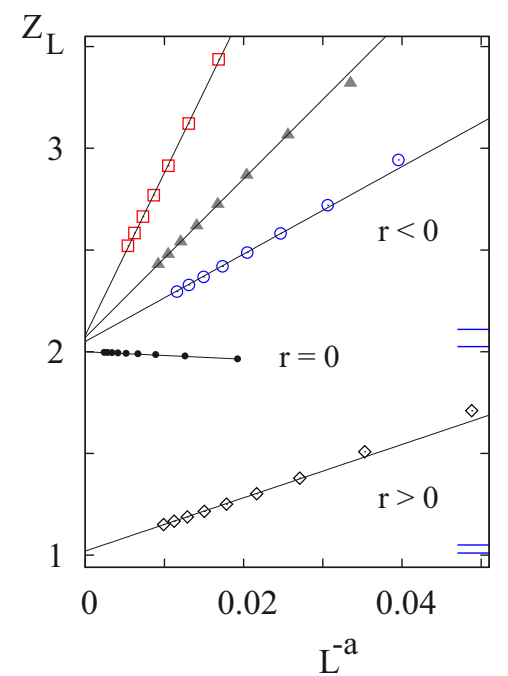

FIG. 5. (Color online) Extrapolations of effective dynamic exponents [Eq. (20)] within the regions $0<r=-J_{2} /\left|J_{1}\right| \leqslant 0.3$ (rhomboids), $r=0$ (dots), $0<-r<1$ (circles), $r=-1$ (triangles), and $r<-1$ (squares). Nonlinear fittings in the weak frustrated regime $(a \sim 1.45)$ yield a dynamic exponent $z \simeq 1.03 \pm 0.02$, while those for circles $(a \sim 1.40)$, triangles $(a \sim 1.47)$, and squares $(a \sim 1.64)$ yield slightly subdiffusive exponents within the confidence interval $[2.02,2.11]$. The latter and that of the weak competing situation are depicted by rightmost horizontal lines. The usual $r=0$ case converges faster $(a \sim 1.95)$ towards the exact exponent within the same range of sizes.

ascribed to our finite-size limitations. In that sense, the merging of confidence intervals (rightmost center of Fig. 5) suggests a common characterization of the three noncompeting branches of Fig. 3 within an error margin of $\sim 4 \%$. On the other hand, for $J_{2} \gg\left|J_{1}\right|$ one should recover the usual diffusive dynamics as the system would then reduce to two independent Glauber processes, thus suggesting a slight overestimation of dynamic exponents in this region. (Note that this case essentially differs from the regime $J_{2} \rightarrow 0^{-}$, because for the latter all kinks separated by two dual spacings strongly repel each other in the low-temperature limit).

\section{CONCLUDING DISCUSSION}

Recapitulating, we have constructed a symmetric spin- $\frac{1}{2}$ representation of the $1 \mathrm{D}$ Glauber dynamics with both firstand second-neighbor interactions by which we analyzed the scaling behavior of relaxation times of finite chains under various situations at infinitesimal temperature. The resulting time scales were read off from the spectral gaps of the corresponding evolution operator built up in Sec. II [Eqs. (11a), (11b), and (15)] to account for the correlated kink pairing and diffusion processes schematized in Table I.

Special attention was paid to weakly frustrated regimes $\left(J_{2}<0\right)$ where energy barriers hindering the coalescence of magnetic domains (second and third diffusion processes of Table I) cause the relaxation time to grow unbounded as $e^{4\left|K_{2}\right|}$, even for finite systems (Fig. 1). However, a clear finite-size scaling regime [Eq. (19)] turned out to take over the low 
but nonzero temperature limit of those time scales, at least within our accessible range of competing interactions [main panels of Fig. 2 and inset of Fig. (2b)]. In stark contrast to the noncoarsening scenario at $T=0[5,15]$, that led us to suggest a nearly ballistic dynamic exponent $z \simeq 1.03(2)$ (lowermost sequence extrapolations of Fig. 5), with which domains coarsen almost linearly in time. Still, due to the mentioned coalescence barriers, they do so at arbitrarily slow velocities $\propto e^{-4\left|K_{2}\right|}$ as suggested by Eq. (19). It would be important to examine also the weakly frustrated region $1 / 2<$ $-J_{2} /\left|J_{1}\right|<1$ where, to reach one of its fourfold degenerate states $(\cdots|\bullet \bullet| \circ \circ \mid \cdots)$, the dynamics must first create kink pairs to break up isolated ferro domains of length $\geqslant 3$ [5]. However, as noted in Sec. III A, in lowering the temperature the progressive smallness of spectral gaps precluded the Lanczos convergence on larger chains; a drawback which already appeared for $-J_{2} /\left|J_{1}\right| \gtrsim 0.3$ and still remains an open issue. On the other hand, analytical approximations in that region would have to arbitrarily decouple the infinite hierarchy of equations of motion embodied in the evolution operator. But in view of the discontinuous scaling behavior obtained even for $J_{2} \rightarrow 0^{-}$, such a procedure might well result inappropriately at low-temperature regimes.

When it comes to noncompeting regimes $\left(J_{2}>0\right)$, the convergence problem no longer emerges as there are no metastable states slowing down the dynamics, thus relaxation times of finite systems are kept bounded even for $T=0$. This facilitated the numerical analysis of spectral gap discontinuities occurring as a result of those that naturally appear in the limit of $T \rightarrow 0$ on the transition probability rates at $J_{2} /\left|J_{1}\right|=0$ and 1 (Fig. 3). In turn, this resulted in three branches of gaps whose typical scaling regimes were initially characterized by somewhat similar dynamic exponents (Fig. 4). The proximity among the latter, however, became much closer after extrapolating the sequence of finite-size effective approximants [Eq. (20)] summarized in Fig. 5 and to the point of suggesting an almost diffusive exponent $\forall J_{2}>0$.

Finally, and with regard to a possible extension of this study, it would be interesting to apply these scaling techniques also to the 1D Kawasaki dynamics [1] (say, for $J_{1}>0$ ), where, due to the strict constancy of magnetization, initial disordered states quenched to zero temperature get stuck in metastable phases already for $J_{2}=0$ [4]. As before, these just involve ferromagnetic domains of two or more spins, the number of such configurations growing as $[(1+\sqrt{5}) / 2]^{L}$. However, for $1 / 2<J_{2} / J_{1}<1$ it can be readily verified that states having two or more consecutive domains of length two would be excluded from that metastable set. That further constraint would still leave us with a number of configurations growing as $\gamma^{L}$, though with $\gamma$ actually smaller than the golden ratio. For infinitesimal temperatures this raises the question of whether such reduction of metastability could also bring about strong changes in the scaling regimes of this phase separation dynamics. Further work in that direction is in progress. As for $d>1$, in principle, a quantum spin representation could also be constructed, though its Lanczos diagonalization would be restricted to only a few and small lattices, thus rendering finite-size scaling impractical.

\section{ACKNOWLEDGMENTS}

I thank H. D. Rosales and F. A. Schaposnik for helpful discussions. Support from CONICET and ANPCyT, Argentina, under Grants No. PIP 2012-0747 and No. PICT 2012-1724, is acknowledged.
[1] For reviews, consult Kinetics of Phase Transitions, edited by S. Puri and V. Wadhawan (CRC Press, Boca Raton, FL, 2009); A. J. Bray, Adv. Phys. 43, 357 (1994); J. D. Gunton, M. San Miguel, and P. S. Sahni, in Phase Transitions and Critical Phenomena, edited by C. Domb and J. L. Lebowitz (Academic Press, London, 1983), Vol. 8.

[2] R. J. Glauber, J. Math. Phys. 4, 294 (1963).

[3] B. U. Felderhof, Rep. Math. Phys. 1, 215 (1971).

[4] V. Spirin, P. L. Krapivsky, and S. Redner, Phys. Rev. E 63, 036118 (2001); 65, 016119 (2001); P. L. Krapivsky, S. Redner, and E. Ben-Naim, A Kinetic View of Statistical Physics (Cambridge University Press, Cambridge, 2010), Chap. 8.

[5] S. Redner and P. L. Krapivsky, J. Phys. A 31, 9229 (1998).

[6] See e.g. K. Tanaka, T. Morita, and K. Hiroike, Prog. Theor. Phys. 77, 68 (1987); R. M. Hornreich, R. Liebmann, H. G. Schuster, and W. Selke, Z. Phys. B 35, 91 (1979); J. Stephenson, Phys. Rev. B 1, 4405 (1970).

[7] R. Liebmann, Statistical Mechanics of Periodic Frustrated Ising Systems, Lecture Notes in Physics (Springer-Verlag, Berlin, 1986), Vol. 251. For one-dimensional aspects, see Chap. 2.

[8] This latter is immaterial as it does not affect the relative spin orientation between second neighbors in the ground state.
[9] R. J. Elliott, Phys. Rev. 124, 346 (1961).

[10] For reviews, consult W. Selke, in Phase Transitions and Critical Phenomena, edited by C. Domb and J. L. Lebowitz (Academic Press, London, 1992), Vol. 15; Phys. Rep. 170, 213 (1988); J. Yeomans, in Solid State Physics, Vol. 41, edited by H. Ehrenreich and D. Turnbull (Academic Press, Orlando, FL 1988).

[11] T. Shirakura, F. Matsubara, and N. Suzuki, Phys. Rev. B 90, 144410 (2014); E. Rastelli, S. Regina, and A. Tassi, ibid. 81, 094425 (2010); M. D. Grynberg and H. Ceva, ibid. 36, 7091 (1987).

[12] L. Bogani, C. Sangregorio, R. Sessoli, and D. Gatteschi, Angew. Chem., Int. Ed. Engl. 44, 5817 (2005); K. Bernot et al., J. Am. Chem. Soc. 128, 7947 (2006); A. Caneschi et al., Europhys. Lett. 58, 771 (2002).

[13] M. G. Pini and A. Rettori, Phys. Rev. B 76, 064407 (2007).

[14] Z. R. Yang, Phys. Rev. B 46, 11578 (1992).

[15] P. Sen and S. Dasgupta, J. Phys. A 37, 11949 (2004); P. Sen and P. K. Das, in Quantum Annealing and Related Optimization Methods, Vol. 679, Lecture Notes in Physics, edited by A. Das and B. K. Chakrabarti (Springer-Verlag, Berlin, 2005).

[16] W. Feller, An Introduction to Probability Theory and Its Applications, 2nd ed., Vol. 2 (Wiley, New York, 1970), Chap. 1. 
[17] In turn for $r>1 / 2$ and $\forall T>0$, the two-spin correlations also exhibit oscillations with a temperature-dependent wavelength; see transfer matrix evaluations of Refs. [6,7,10].

[18] P. C. Hohenberg and B. Halperin, Rev. Mod. Phys. 49, 435 (1977).

[19] Consult, for instance, M. Henkel, H. Hinrichsen, and S. Lübeck, Non-Equilibrium Phase Transitions, Vol. 1 (Springer, Dordrecht, 2008), Appendix F.

[20] N. G. van Kampen, Stochastic Processes in Physics and Chemistry, 3rd ed. (North-Holland, Amsterdam, 2007), Chap. 5.

[21] See, for example, G. H. Golub and C. F. van Loan, Matrix Computations, 3rd ed. (Johns Hopkins University Press, Baltimore, 1996), Chap. 9; L. Komzsik, The Lanczos Method: Evolution and Application (SIAM, Philadelphia, 2003).
[22] A. J. Guttmann, in Phase Transitions and Critical Phenomena, edited by C. Domb and J. L. Lebowitz (Academic Press, London, 1989), Vol. 13.

[23] G. M. Schütz, in Phase Transitions and Critical Phenomena, edited by C. Domb and J. L. Lebowitz (Academic Press, London, 2001), Vol. 19; D. C. Mattis and M. Lawrence Glasser, Rev. Mod. Phys. 70, 979 (1998).

[24] See Gershgorin theorem in I. S. Gradstheyn and I. M. Ryzhik, Tables of Integrals, Series, and Products, 5th ed. (Academic Press, New York, 1994), Chap. 15.

[25] J.-M. Vanden Broeck and L. W. Schwartz, SIAM J. Math. Anal. 10, 658 (1979); C. J. Hamer and M. N. Barber, J. Phys. A 14, 2009 (1981). 